\title{
Autophagic death induced by thermo-chemotherapy in gastric cancer cells results from the reactive oxygen species pathway
}

\author{
YINBING WU ${ }^{1}$, MINGXIN PAN $^{2}$, SHUZHONG CUI $^{1}$, MINGCHEN BA $^{1}$, \\ ZULONG CHEN $^{1}$ and QIANG RUAN ${ }^{1}$ \\ ${ }^{1}$ Treatment Center of Body Cavitary Thermo-perfusion, Cancer Hospital of Guangzhou Medical University, \\ Guangzhou, Guangdong 510095; ${ }^{2}$ Second Department of Hepatobiliary Surgery, Zhujiang Hospital, \\ Southern Medical University, Guangzhou, Guangdong 510282, P.R. China
}

Received May 13, 2015; Accepted February 25, 2016

DOI: $10.3892 / \mathrm{mmr} .2016 .5353$

\begin{abstract}
Gastric cancer is the third leading type of cancer and has the third leading cancer-associated mortality in China. The mechanism of thermo-chemotherapy in gastric cancer cells remains to be elucidated. The present study aimed to investigate the role of autophagic cell death in the thermo-chemotherapy of gastric cancer. The current study included four groups: An empty control group, a hyperthermia group, a chemotherapy (oxaliplatin) group, and a thermo-chemotherapy group. Cell viability was analyzed by the MTS assay. Production of intracellular reactive oxygen species (ROS) was quantified by flow cytometry. Autophagy-associated proteins, Beclin 1, microtubule-associated protein 1A/1B-light chain (LC3B) and mammalian target of rapamycin (mTOR), were determined by western blot analysis. The results indicated that thermo-chemotherapy markedly increased intracellular ROS production, and decreased mitochondrial membrane potential. The transmission electron microscopy results indicated that thermo-chemotherapy induced production of autophagic bodies. In addition, thermo-chemotherapy-induced cell damage at the cellular and animal levels indicated a notable increase in the expression of the autophagy-associated genes, LC3B and Beclin 1. A negative correlation between mTOR expression and autophagy was also identified, which demonstrates that thermo-chemotherapy induces autophagic
\end{abstract}

Correspondence to: Professor Mingxin Pan, Second Department of Hepatobiliary Surgery, Zhujiang Hospital, Southern Medical University, 253 Middle of Gongye Avenue, Guangzhou, Guangdong 510282, P.R. China

E-mail: pmxwxxyy@yeah.net

Professor Shuzhong Cui, Treatment Center of Body Cavitary Thermo-perfusion, Cancer Hospital of Guangzhou Medical University, 78 Hengzhigang Road, Guangzhou, Guangdong 510095, P.R. China

E-mail: cuishuzhong@126.com

Key words: gastric cancer, thermo-chemotherapy, hyperthermic intraperitoneal chemotherapy, reactive oxygen species, autophagy cell death by activating the autophagy-associated signaling pathways. The results of the present study demonstrated that the ROS level is important in autophagic death of the gastric carcinoma cells, and the increased ROS level, induced by thermo-chemotherapy treatment, induced autophagy in gastric carcinoma cells.

\section{Introduction}

Gastric cancer is the third leading type of cancer and has the third leading cancer-associated mortality in China. The prognosis of gastric cancer is generally poor and, due to the development pattern and the characteristics of gastric cancer, the majority of patients exhibit peritoneal metastasis upon diagnosis or develop peritoneal carcinomatosis following surgery (1-3). Targeted prevention and treatment of peritoneal carcinomatosis from gastric cancer is important to improve patients' quality of life and prognosis. Recent studies have demonstrated that hyperthermic intraperitoneal chemotherapy (HIPEC) exhibits increased efficacy in prevention and treatment of peritoneal carcinomatosis from gastric cancer $(4,5)$, however, the underlying molecular mechanisms remain to be elucidated.

Due to the lower heat resistance of tumor cells compared with normal cells, thermo-chemotherapy eradicates tumor cells with increased efficacy using high temperature and increased drug sensitivity in the cancer cell population. Autophagy is important in apoptosis induced by thermo-chemotherapy and studies have demonstrated that, under different conditions, autophagy may either result in tumor cell death or promote tumor cell survival (6). Following physicochemical damage, including radiation therapy and chemotherapy, increased levels of basal autophagy improves the ability of tumor cells to endure and repair damage (6). However, excessive autophagy in tumor cells promotes autophagic cell death, resulting in an effective treatment of cancer (7). Thus, in recent years, induction of autophagic cell death has been considered an important cancer therapeutic strategy.

Studies have demonstrated that reactive oxygen species (ROS) induce the dissociation of autophagy molecules, Beclin 1 and B-cell lymphoma 2 (Bcl-2), which activates Beclin 1 and stimulates autophagy pathways, thus leading 
to the inhibition of mammalian target of rapamycin (mTOR) and an increased expression of the autophagy marker, LC3 II. The subsequent activation of autophagy-associated signaling pathways induce autophagic cell death (8). A recent study demonstrated that thermo-chemotherapy induces oxidative stress and increases ROS levels in tumor cells (9). A combination of thermo-chemotherapy and oxidative stress inducer, tert-butyl hydroperoxide increases intracellular ROS levels and improves the cytotoxicity against tumor cells (10). Thus, the stimulation of ROS production and the induction of tumor cell apoptosis may be a major anti-tumor mechanism of thermo-chemotherapy.

The present study hypothesizes that one of the major mechanisms of thermo-chemotherapy is to increase ROS levels and stimulate oxidative stress in tumor cells, leading to autophagic death in gastric cancer cells. To investigate this hypothesis, cell culture experiments and animal models were used. Thermo-chemotherapy was simulated, and the correlation between the production of ROS and the expression of autophagy-associated genes in gastric cancer cells was examined. In addition, the effect of thermo-chemotherapy-induced ROS production on autophagic cell death was investigated. The role of ROS-induced autophagic cell death in the cytotoxic effect of thermo-chemotherapy in gastric cancer cells was further investigated. The present study may elucidate the mechanisms of HIPEC in the treatment and prevention of gastric cancer peritoneal metastasis.

\section{Materials and methods}

Identification of the half-maximal inhibitory concentration $\left(I_{50}\right)$ of oxaliplatin ( $\left.\mathrm{L}-\mathrm{OHP}\right)$. The SGC-7901 human gastric carcinoma cell line was treated with L-OHP. When the SGC-7901 cells entered the exponential phase, the L-OHP was added to Dulbecco's modified Eagle's medium (Hyclone, Logan, UT, USA) at various concentrations $(10,20,40,80,160$ and $320 \mu \mathrm{g} / \mathrm{ml})$. Following L-OHP treatment for $24 \mathrm{~h}$, cell viability was determined using the MTS assay to determine the most effective concentration of L-OHP The protocol for the MTS assay was performed according to a previous study (10). MTS and dimethyl sulfoxide used in the MTS assay were purchased from Sigma-Aldrich (St. Louis, MO, USA). The $\mathrm{IC}_{50}$ was obtained from three independent experiments, and was used in the following studies.

Treatment of cells with L-OHP and hyperthermia (HT). SGC-7901 cells were treated with the most effective L-OHP concentration for $1 \mathrm{~h}$, then the cells were exposed to different temperatures $\left(39,41,42,43\right.$ and $\left.45^{\circ} \mathrm{C}\right)$ for different assays. Following incubation for $24 \mathrm{~h}$, cell viability was monitored using the MTS assay to determine the most effective temperature.

Grouping and treatment of the cells. To ensure the most effective concentration of L-OHP and use of the optimum temperature, SGC-7901 cells were randomly divided into four groups, including: Group 1, the control group; group 2: the HT group, treated with HT at the optimum temperature; group 3 , chemotherapy group, treated with L-OHP using the most effective concentration; and group 4, chemotherapy + HT group, treated with most effective concentration of L-OHP and HT. The cells cultured at $37^{\circ} \mathrm{C}$ served as the negative control group.

Establishment of gastric cancer model. To establish a gastric cancer model, $30 \mathrm{BALB} / \mathrm{c}-\mathrm{nu}$ nude mice (pathogen-free grade; age, 4-6 weeks old; weight, 230-280 g; purchased from the Animal Center of the Guangzhou Medical University, Guangzhou, China) were used in the present study. The mice were maintained in a pathogen-free environment with a $12 \mathrm{~h}$ light/dark cycle, and were given adequate nutrition and water. This study was approved by the ethics committee of Southern Medical University (Guangzhou, China). SGC-7901 cells were subcutaneously injected into each side of the posterior flank groin region of the mouse. The nude mice were randomly divided into four groups $(n=3)$ : Group 1, the control group, in $37^{\circ} \mathrm{C}$, injected intraperitoneally with glucose; group 2 , HT group, in $43^{\circ} \mathrm{C}$, injected intraperitoneally with glucose (6 mg/kg, Sigma-Aldrich); group 3, chemotherapy group, local intraperitoneal injection to the tumor at a dose of $6 \mathrm{mg} / \mathrm{kg}$ L-OHP; and group 4, chemotherapy + HT group, injected intraperitoneally at a dose of $6 \mathrm{mg} / \mathrm{kg} \mathrm{L}-\mathrm{OHP}$ and exposed to $43^{\circ} \mathrm{C}$. The drug sensitivity of cells to L-OHP was determined using the Eppendorf BioSpectrometer (Eppendorf, Hamburg, Germany). The optical density value represents the sensitivity of the cells. Following administration of chemotherapy or glucose for $1 \mathrm{~h}$, the mice were fixed in a water box. The water was preheated to $43^{\circ} \mathrm{C}$ and the depth of the water in the box kept the tumor region immersed in the water. The box was placed into a biochemical incubator (JYSP-450; Hefei Jayon Instrument Equipment Co. Ltd., Hefei, China) at $43^{\circ} \mathrm{C}$ for $1.5 \mathrm{~h}$, to ensure the tumor cells were heated for at least $1 \mathrm{~h}$.

Western blot analysis. The expression levels of autophagy-associated proteins, Beclin 1, LC3B and mTOR were detected by western blotting. The treatment of trigeminal ganglion and the western blot processes were performed according to the previous study (10). In this study, mouse anti-Beclin 1 (cat. no. sc-48341), anti-LC3 $\beta$ (cat. no. sc-271625) and antimTOR (cat. no. sc-293089) monoclonal antibodies were used Santa Cruz Biotechnology, Inc. (Santa Cruz, Dallas, TX, USA; 1:2,000). The horseradish peroxidase-conjugated rabbit anti-mouse antibody (cat. no. sc-358920; Santa Cruz Biotechnology, Inc.; 1:1,000) was used as the secondary antibody.

Measurement of parameters. The mice used in the gastric cancer models were sacrificed by cervical dislocation following treatment according to the conditions of each group for $24 \mathrm{~h}$. Prior to sacrifice, mice were anaesthetized with pentobarbital sodium (1 mg/kg, Tiangen Biotech (Beijing) Co., Ltd., Beijing, China). The tumor tissue $(n=10)$ was surgically removed and part of the tumor tissue was incubated in collagenase IV (Sigma-Aldrich) to isolate single tumor cells to assess the level of ROS in the cells by flow cytometry. MMP and apoptosis were also analyzed by flow cytometry (17548; Beckman Coulter, Brea, CA, USA). The protocol of the flow cytometetry was performed according to a previous study (10). Another part of the tumor tissue was fixed with $10 \%$ neutral-buffered formalin (Sigma-Aldrich), embedded in paraffin and cut into $5-\mu \mathrm{m}$ sections with a microtome (M3500; Labway Science 
Development Ltd., Beijing, China). The expression levels of autophagy-associated proteins, Beclin 1, LC3B and mTOR, were examined by immunohistochemistry according to a previous study (10). The same antibodies were used as in the western blot analysis section.

Transmission electron microscopy analysis. The cells grew on the microcarriers and observed before treatment. Microcarriers were placed in a mixture of (1:1) propylene oxide/Epon resin (Sigma-Aldrich). Then, the microcarriers were left overnight in pure resin for impregnation of the cells. The microcarriers were embedded in the Epon resin (Sigma-Aldrich). The ultra-thin sections were obtained with a Leica EM UC7 ultramicrotome (Leica, Wetzlar, Germany). Sections were deposited on the formvar/carbon-coated nickel grids and stained with 5\% uranyl acetate and 5\% lead citrate. The cells were observed using a JEOL 1011 transmission electron microscope (TEM; JEOL Ltd., Tokyo, Japan).

Statistical analysis. Statistical analysis was performed using Origin 6.0 and SPSS 19.0 software package (IBM SPSS, Armonk, NY, USA). Continuous data were expressed as the mean \pm standard deviation. One-way analysis of variance (ANOVA) was used to examine statistically significant differences between groups. Least significant difference was used for multiple comparisons and factorial design ANOVA was used for factorial design data. The effect of combination therapy of L-OHP and HT on SGC-7901 cell viability was analyzed using the q-value method (11). P<0.05 (two-tailed) was used to indicate a statistically significant difference.

\section{Results}

L-OHP treatment inhibits SGC-7901 cell viability. The result of the MTS assay indicated that the different concentrations of L-OHP inhibited SGC-7901 cell viability. As the L-OHP concentration increased, the SGC-7901 cell viability was significantly decreased, and there was a positive correlation between L-OHP concentration and inhibition of cell viability (Fig. 1A). Statistical analysis of the results demonstrated that the cell viability in the eight groups was significantly different $(\mathrm{F}=1,598.325, \mathrm{P}=0.05)$. The cell viability was also significantly different between any two groups (Fig. 1B; $\mathrm{P}<0.05$ ).

Sensitivity of SCG-7901 cells to L-OHP correlates with $\mathrm{IC}_{50}$ regression. According to the results of the MTS assay, the inhibitory rate was obtained from different concentrations of L-OHP. These results indicated that the drug sensitivity of SGC-7901 cells was positively correlated with the L-OHP inhibition rate (Fig. 2A). Furthermore, the drug sensitivity of the SCG-7901 cells was also correlated with the $\mathrm{IC}_{50}$ regression curve (Fig. 2B; $\mathrm{P}<0.05, \mathrm{r}=0.7832$ ).

The results indicated that the $\mathrm{IC}_{50}$ was $80.66 \mu \mathrm{g} / \mathrm{ml}$ (Fig. 2B), and therefore a concentration of L-OHP of $80 \mu \mathrm{g} / \mathrm{ml}$ was used in the subsequent experiments.

HT treatment inhibited SGC-7901 cell viability. To investigate the effect of temperature on the SGC-7901 cell viability, the SGC-7901 cells were treated with different temperatures $\left(37,39,41,42,43\right.$ and $\left.45^{\circ} \mathrm{C}\right)$ when the cells entered the exponential phase. Following treatment with $80 \mu \mathrm{g} / \mathrm{ml}$ L-OHP for $24 \mathrm{~h}$, the SGC-7901 cells were heated for $1 \mathrm{~h}$ and cultured for a further $24 \mathrm{~h}$. Cell viability was monitored using the MTS assay (Fig. 3A). The results indicate that the cell viability was gradually decreased following the increase in temperature and that high temperature resulted in cell death in an increased number of the tumor cells. Statistical analysis of the results demonstrated that the interaction between L-OHP and temperature was significant in the suppression of cell proliferation (Fig. 3B; $F=25,201.956, P=0.05 ; F=993.630$, $\mathrm{P}=0.05)$.

L-OHP and HT inhibit SGC-7901 cell viability. According to the results of the $\mathrm{IC}_{50}$ determination and the optimum temperature experiments, a concentration of $80 \mu \mathrm{g} / \mathrm{ml}$ $\mathrm{L}-\mathrm{OHP}$ and $43^{\circ} \mathrm{C}$ were selected for the incubation of the SGC-7901 cells, and to demonstrate the synergistic effect. The cell viability was analyzed using the MTS assay. Compared with the control group, the cell viability of the HT group, chemotherapy group and chemotherapy + HT group were significantly decreased $(\mathrm{P}<0.05)$. The reduced cell viability in the chemotherapy $+\mathrm{HT}$ group was the highest $(\mathrm{P}<0.01$; Fig. 4A).

Furthermore, the cells were observed under an inverted microscope (model, IX73; Olympus, Tokyo, Japan). Compared with control group, the cell density of the HT and chemotherapy groups were reduced and the cells were observed. Cell growth in the chemotherapy + HT group was slowest, the cells were observed to be round and floating in the medium, and cell apoptosis occurred to the greatest extent in this group (Fig. 4B).

The effect of combination therapy of L-OHP and HT for SGC-7901 cell viability was calculated by the q-value method. The q-value was determined to be 1.370 , suggesting that the combination therapy of L-OHP and HT had a synergistic effect to inhibit cell viability.

Combination therapy of $\mathrm{L}-\mathrm{OHP}$ and $H T$ promotes expression of ROS. The ROS level was detected by flow cytometry. Compared with the control group, the ROS level of the HT, chemotherapy and chemotherapy + HT groups were significantly increased (Fig. 5A; $\mathrm{P}<0.01$ ). The increase in ROS level was highest in the chemotherapy + HT group (Fig. 5B; $\mathrm{P}<0.01)$.

Combination of L-OHP and HT reduces SGC-7901 cell mitochondrial membrane potential (MMP) and promotes SGC-7901 cell apoptosis. The MMP was analyzed by flow cytometry. Compared with the control group, the MMP of the HT, chemotherapy and chemotherapy + HT groups were decreased. The MMP level of the chemotherapy group and the chemotherapy + HT group were significantly decreased (Fig. 6A; $\mathrm{P}<0.01$ ).

The apoptosis of SGC-7901 cells was also detected by flow cytometry. Compared with the control group, the HT group had no significant effect on the apoptosis of SGC-7901 cells, which demonstrated that HT only inhibited the cell viability, with no effect on the apoptosis of SGC-7901 cells. However, treatment with L-OHP or L-OHP + HT significantly increased apoptosis in SGC-7901 cells (Fig. 6B; $\mathrm{P}<0.01$ ). 

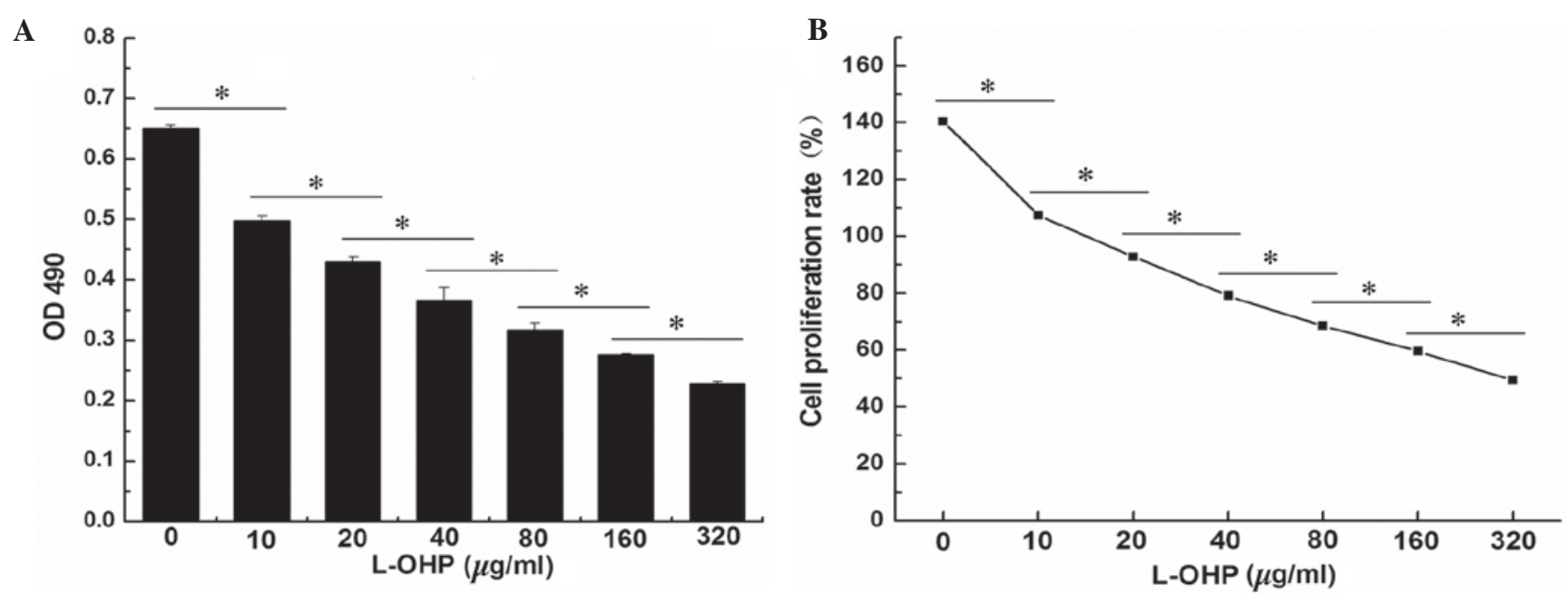

Figure 1. L-OHP sensitivity of SGC-7901 cells and the proliferation rate at various concentrations. (A) Drug sensitivity of cells to L-OHP. (B) Cell proliferation rate at different concentrations of L-OHP. "P<0.05 vs. the previous concentration of L-OHP. OD, optical density; L-OHP, oxaliplatin. OD, optical density.

Combination therapy effect of $L-O H P+H T$ resulted in autophagic cell death. In order to investigate whether the apoptosis induced by the L-OHP + HT treatment was associated with autophagy, the autophagosome produced was observed under a transmission electron microscope (5656AG; FEI, Eindhoven, The Netherlands) (Fig. 7). The results demonstrate that the cells exhibited greater chromatin pyknosis and autophagosomes in the L-OHP and chemotherapy + HT groups, and the cell membrane appeared incomplete and the cells appeared close to cell death in the chemotherapy + HT group, suggesting the lethal effect of combination therapy with chemotherapy + HT were associated with autophagy.

Combination therapy with $\mathrm{L}-\mathrm{OHP}+\mathrm{HT}$ promotes expression of autophagy-associated proteins. The expression levels of autophagy-associated proteins, Beclin 1, LC3B and mTOR were detected by western blotting. The expression of Beclin 1 and LC3B was demonstrated to be significantly increased in the $\mathrm{L}-\mathrm{OHP}$ and the L-OHP + HT groups $(\mathrm{P}<0.01)$, and the expression of mTOR exhibited a negative correlation with the degree of autophagy, which demonstrated that autophagy-associated protein, LC3, was activated by type I translation into type II, the expression of Beclin 1 was significantly increased, and the expression of mTOR was significantly decreased in the L-OHP and the chemotherapy + HT groups. The results demonstrate that cell death was associated with autophagy. Statistical analysis of the results indicates that the interaction between L-OHP and temperature was significant in the suppression of cell proliferation (Fig. 8; $\mathrm{P}<0.01$ ).

\section{Discussion}

Gastric cancer is important as it has a high incidence and the early symptoms of gastric cancer are easily confused with gastritis or other benign diseases of stomach, making it difficult to diagnose at an early stage when tumors are confined to the mucosa or submucosa. In China, the detection rate of early gastric cancer is only $\sim 5-10 \%$, and the majority of patients are diagnosed at an advanced stage $(2,3,12)$. Diffuse peritoneal carcinomatosis often occurs in patients with serosal invasion. Patients who undergo radical resection may still develop local recurrence, and peritoneal carcinomatosis with malignant ascites is often developed quickly following surgery, leading to poor quality of life for the patients and low long-term survival rates (13). Improving the long-term survival in patients with advanced-stage gastric cancer, and increasing their quality of life, are urgent clinical issues requiring attention.

HIPEC is a novel adjunct treatment for peritoneal carcinomatosis. A large volume of thermo-chemotherapy liquid increases the exposure of peritoneal micrometastases to the chemotherapeutic agents, and maintains high, constant and lasting therapeutic agent concentrations in the abdominal cavity. Thus, HIPEC improves the effect of treatment of peritoneal micrometastases from gastric cancer and peritoneal carcinomatosis-induced ascites (14). Previous clinical studies have demonstrated the effectiveness of HIPEC in treating refractory gastric cancer with malignant ascites (15-17). Currently, HIPEC has been widely used in the treatment of malignant ascites, but the treatment protocol remains under debate. The underlying molecular mechanisms of its therapeutic activity requires elucidation, thus, thermo-chemotherapy cannot be optimized according to these mechanisms. The overall treatment outcomes of peritoneal carcinomatosis from gastric cancer is poor, initiating controversy over the continued use of HIPEC in clinical practice.

In the present study, by establishing an in vitro model for assessing the effect of thermo-chemotherapy using the human gastric cancer cell line SGC-7901, the synergistic effect between the L-OHP and HT was demonstrated. In order to determine the optimal concentration of L-OHP, SGC-7901 cells were treated with different concentrations of L-OHP, and the cell viability in each group was assessed by MTS assay. The results demonstrated that different concentrations of L-OHP inhibited the proliferation of SGC-7901 cells. The inhibition of the cell viability was positively correlated with the concentration of L-OHP, as an increased L-OHP concentration led to increased inhibition of cell viability. 

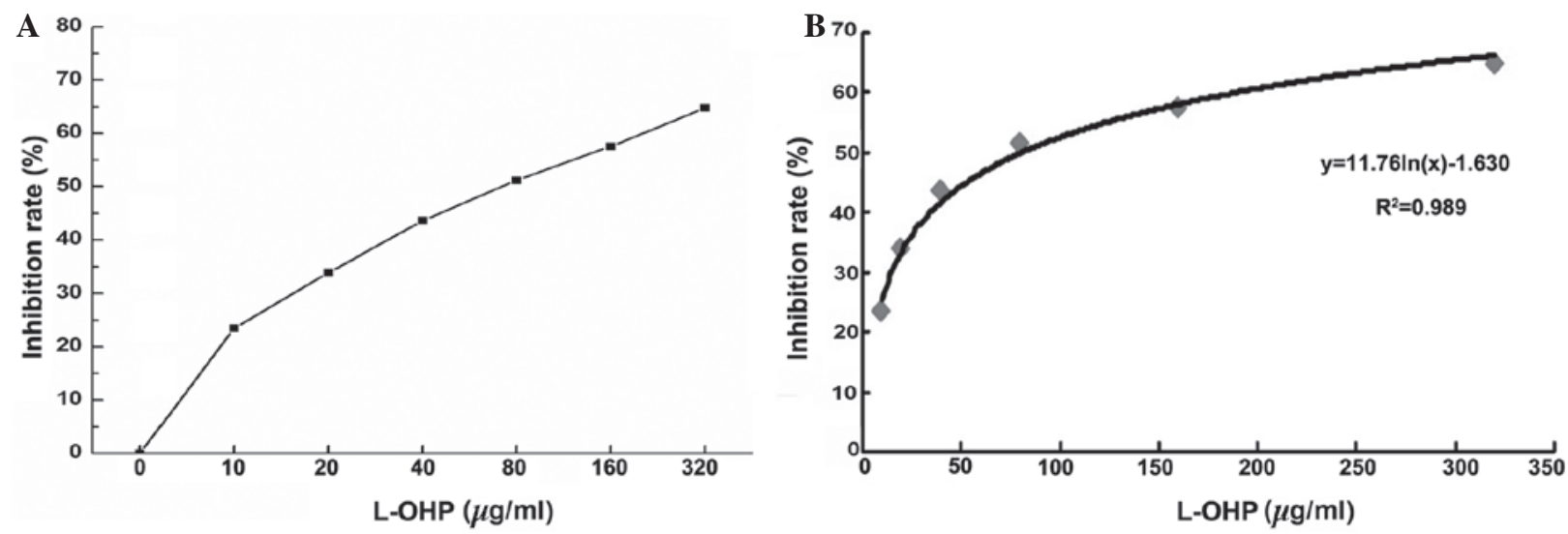

Figure 2. Association between the L-OHP sensitivity of SGC-7901 cells and the L-OHP inhibition rate, or $\mathrm{IC}_{50}$ regression curve. (A) Association between L-OHP sensitivity of SGC-7901 and the L-OHP inhibition rate. (B) Correlation between L-OHP sensitivity of SGC-7901 cells and IC $_{50}$ regression. L-OHP, oxaliplatin; $\mathrm{IC}_{50}$, half-maximal inhibitory concentration.

A

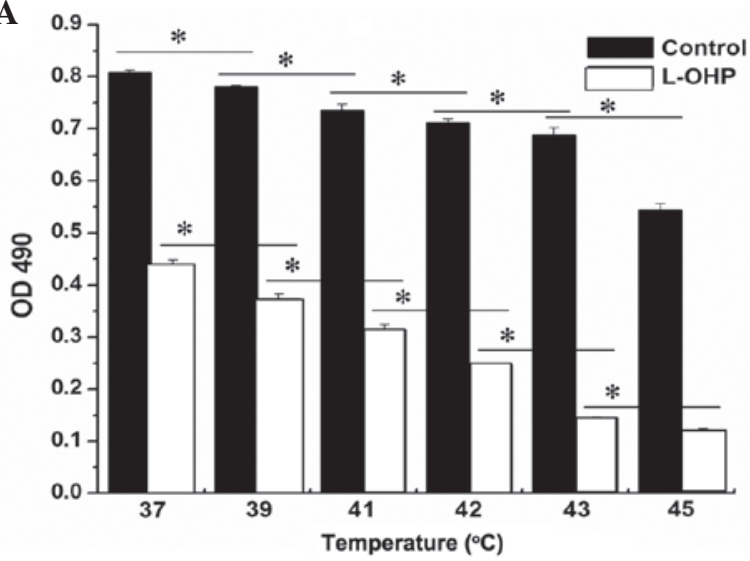

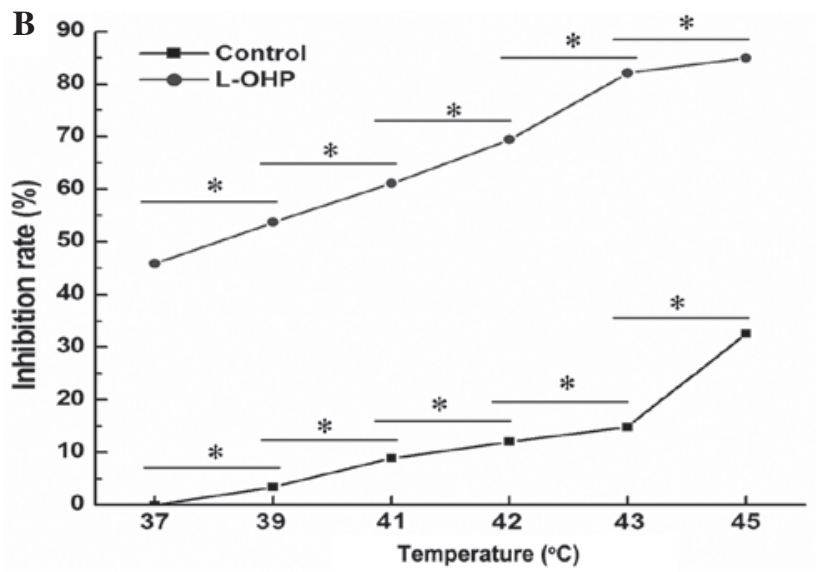

Figure 3. Hyperthermia treatment affects the SGC-7901 inhibition rate. (A) Effects of hyperthermia treatment on the OD value. (B) Effects of hyperthermia on inhibition rate. ${ }^{*} \mathrm{P}<0.05$ vs. the previous temperature. OD, optical density.

A

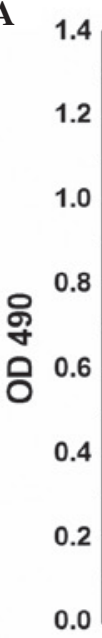

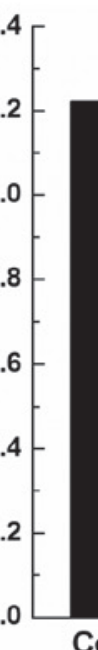
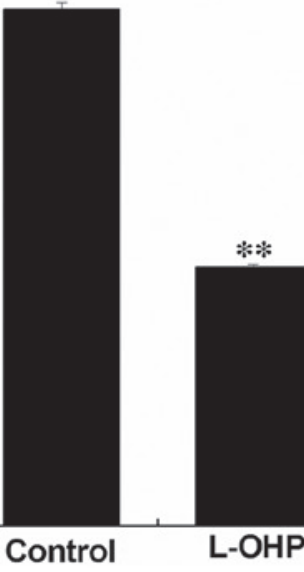

B

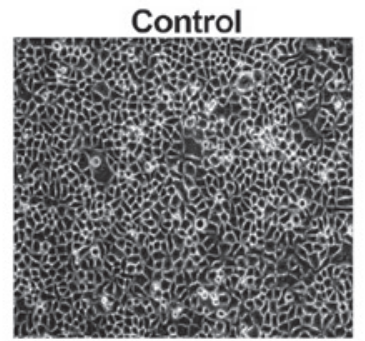

L-OHP

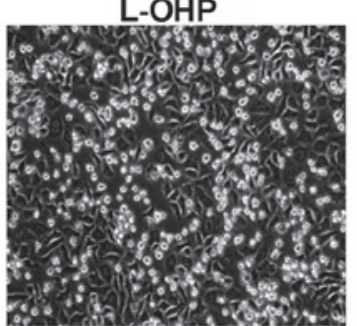

$43^{\circ} \mathrm{C}$

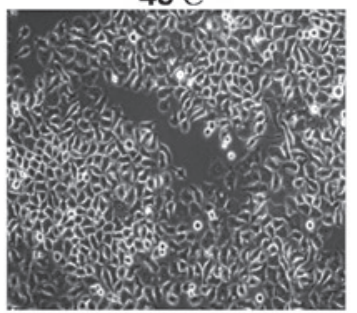

$\mathrm{L}-\mathrm{OHP}+43^{\circ} \mathrm{C}$

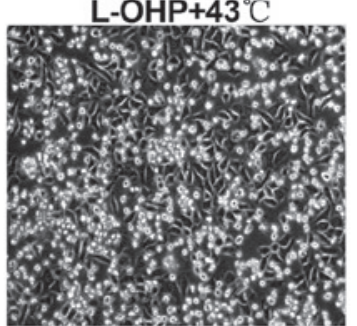

Figure 4. Effects of L-OHP and hyperthermia on SGC-7901 cell viability and morphology. (A) OD value of SGC-7901 cells following different treatments (B) SGC-7901 cell morphology for apoptosis. ${ }^{* *} \mathrm{P}<0.01$ vs. the control group. L-OHP, oxaliplatin; OD, optical density.

The cell viability in each group $\left(37,39,41,42,43\right.$ and $\left.45^{\circ} \mathrm{C}\right)$ was assessed using the MTS assay. The results demonstrated decreased viability with increasing temperature, indicating a cytotoxic effect of hyperthermia. The q-value method (11) 

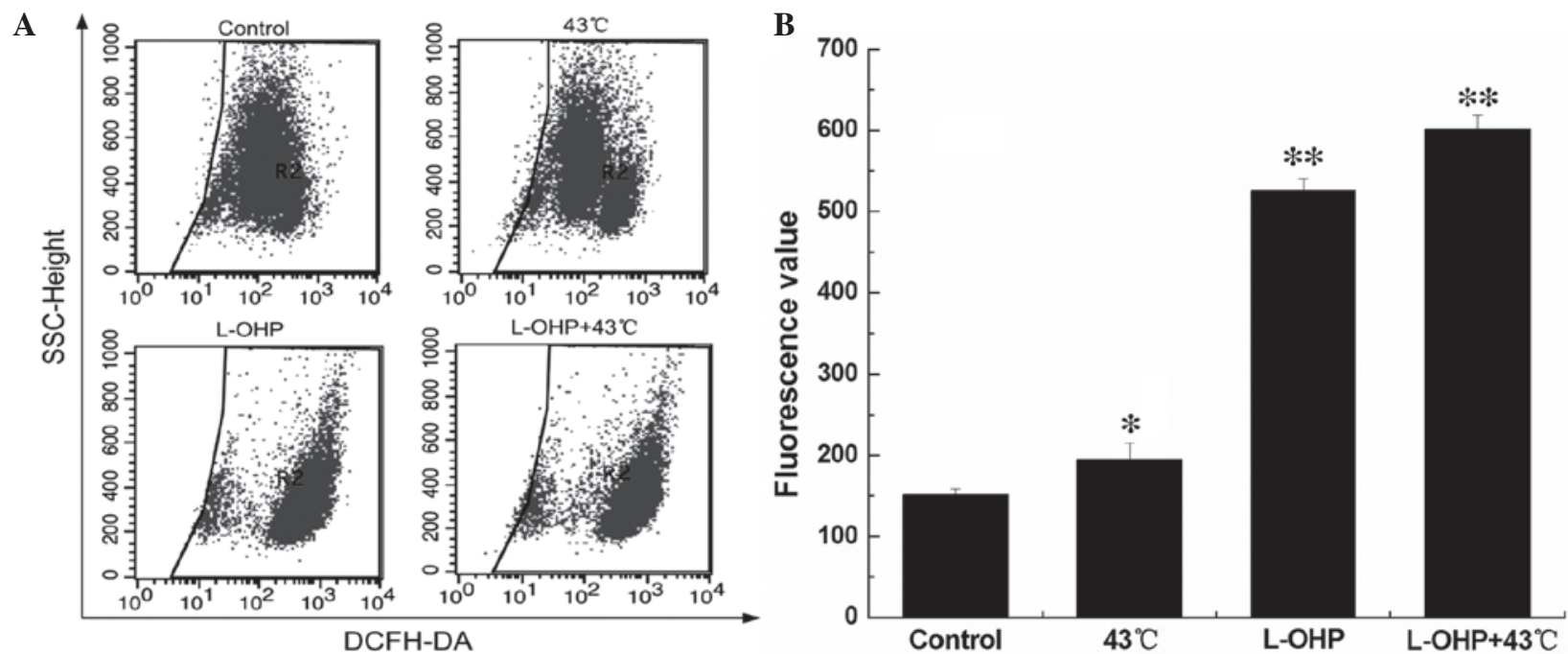

Figure 5. Detection of intracellular ROS expression. (A) ROS expression detected by flow cytometry. (B) Statistical analysis of ROS expression. "P<0.05, ${ }^{* *} \mathrm{P}<0.01$ vs. the control group. ROS, reactive oxygen species; L-OHP, oxaliplatin; DCFH-DA, dichloro-dihydro-fluorescein diacetate.
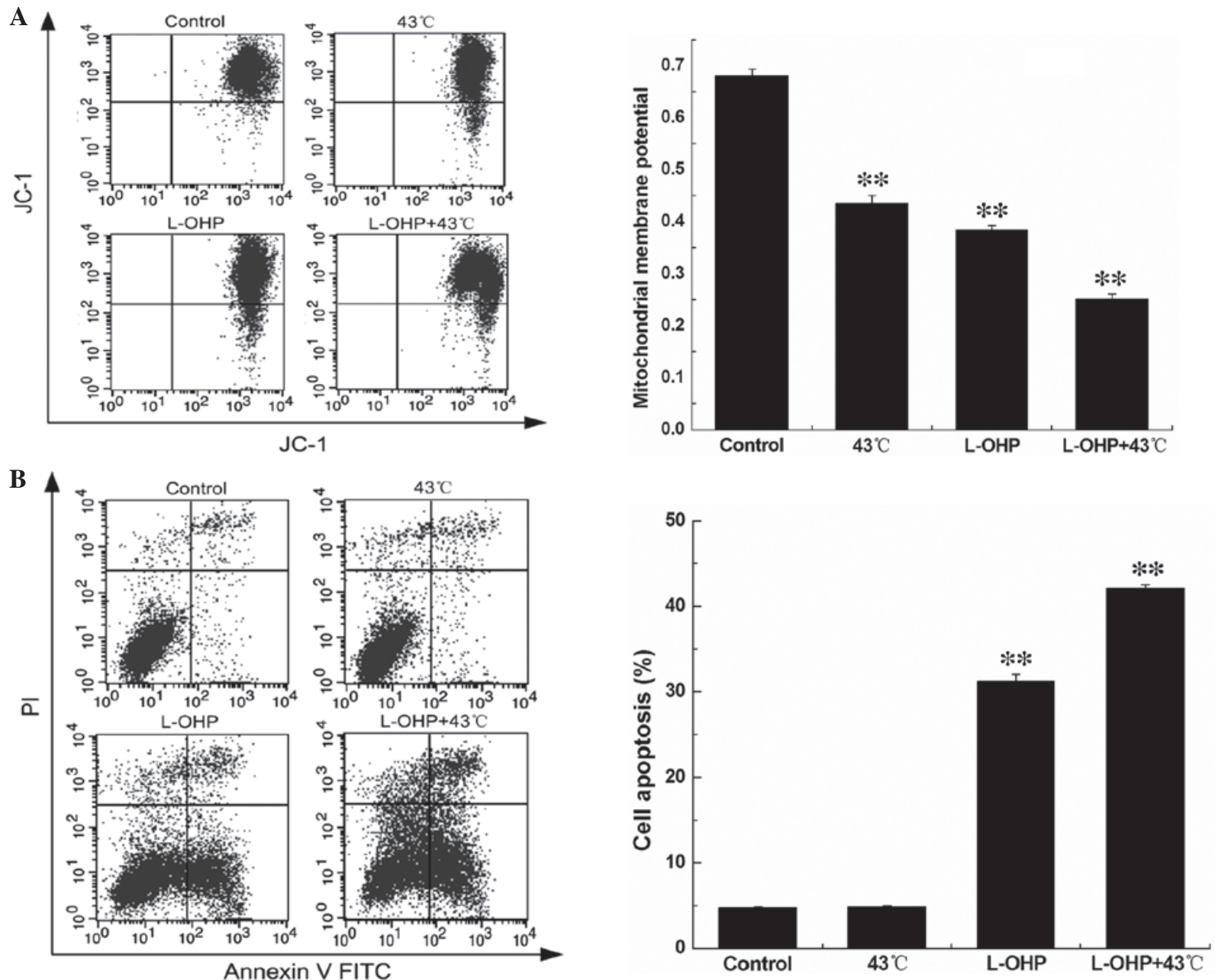

Figure 6. Observation of the SGC-7901 cell mitochondrial membrane potential and apoptosis. (A) Flow cytometric detection of mitochondrial membrane potential in SGC-7901 cells. (B) Apoptosis of SGC-7901 cells detected by flow cytometry. ${ }^{* *} \mathrm{P}<0.01$ vs. the control group. L-OHP, oxaliplatin; PI, propidium iodide.

was used to determine whether there was a synergistic effect between the chemotherapy and hyperthermia. The results demonstrated that at $41^{\circ} \mathrm{C}$, hyperthermia acted synergistically with chemotherapy, and with the rising temperature, 

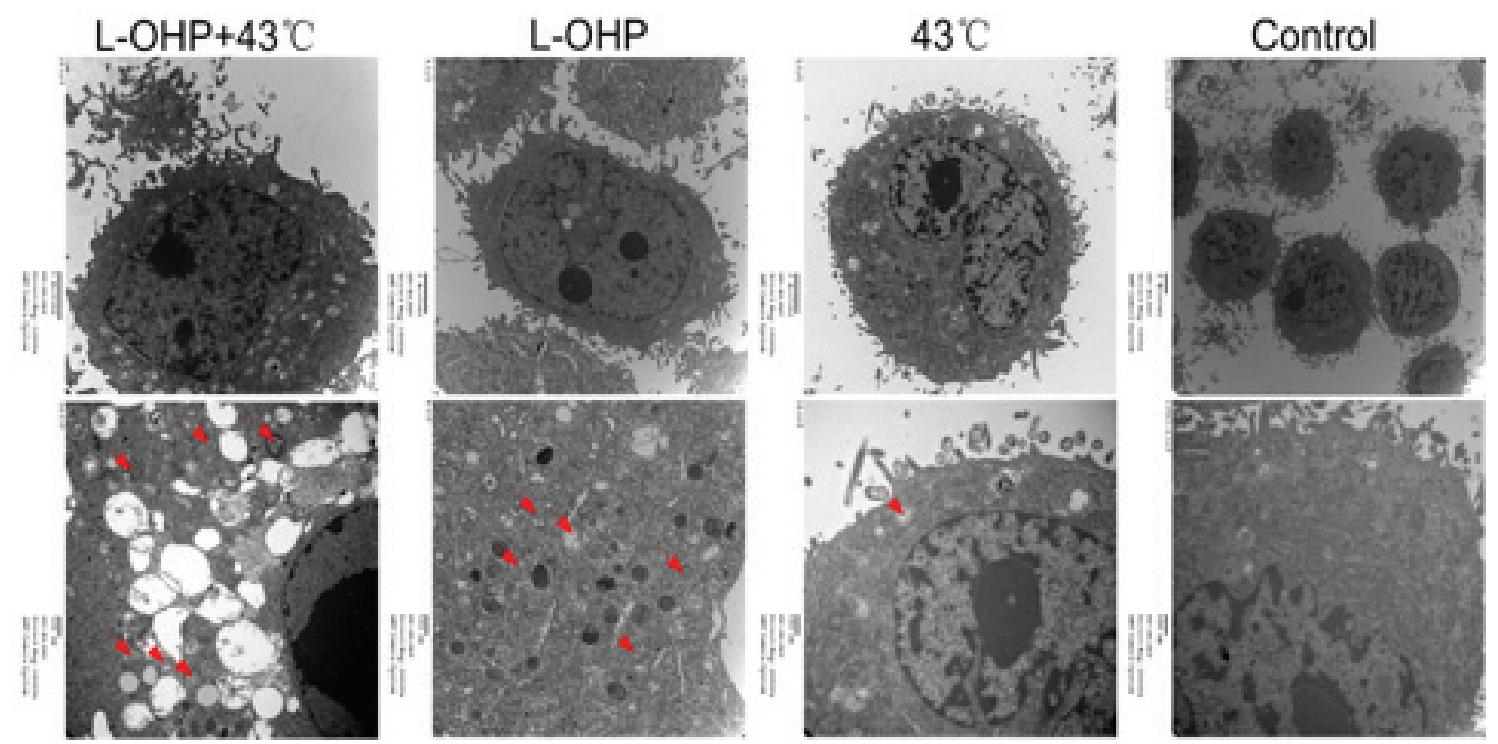

Figure 7. Observation of the autophagic cell death using a transmission electron microscope. Red arrows represent autophagic cells.

A

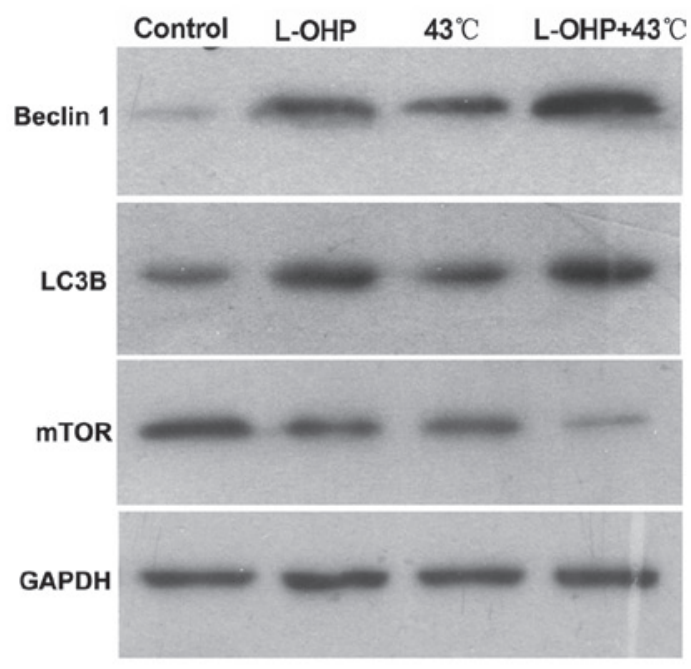

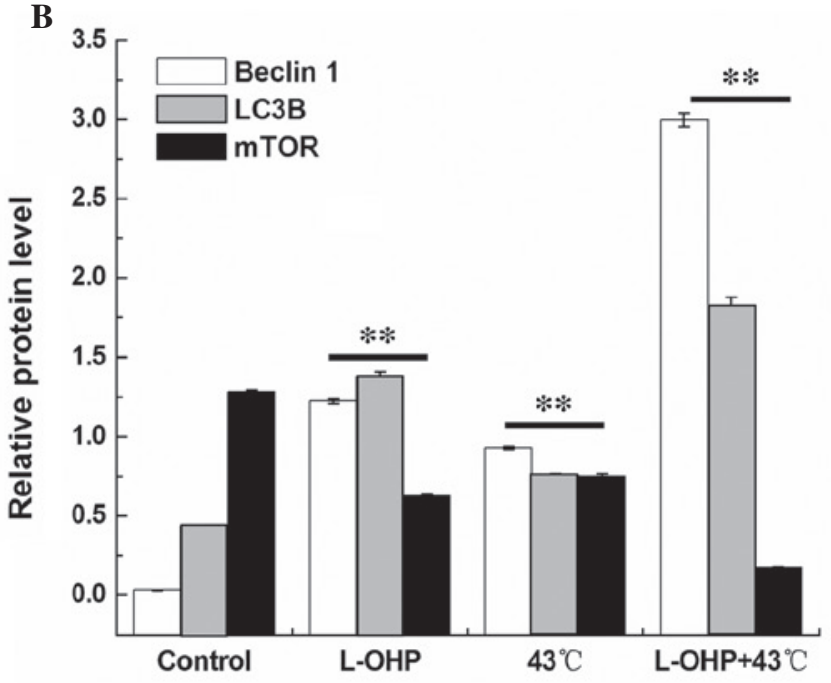

Figure 8. Expression of autophagy-associated proteins. (A) Western blot analysis for autophagy-associated protein expression. (B) Statistical analysis of protein expression. " P<0.01 vs. the control group.mTOR, mammalian target of rapamycin; L-OHP, oxaliplatin; GAPDH, glyceraldehyde-3-phosphate dehydrogenase.

the inhibitory effect on cell proliferation increased; however, from $43-45^{\circ} \mathrm{C}$, the inhibitory effect of hyperthermia on cell proliferation did not change markedly, indicating that thermo-chemotherapy exerted a notable cytotoxic effect on SGC-7901 human gastric carcinoma cells at high temperatures of $43-45^{\circ} \mathrm{C}$. High temperature in the peritoneal cavity may result in thermal damage to the bowel, or intestinal necrosis in severe cases. It also increases the risk of adhesive small bowel obstruction (18). Cui et al (18) used domestic swine as experimental animals and established an animal model of HIPEC using the BR-TRG-I body cavity hyperthermic perfusion extracorporeal circulatory system. Settings of 44 and $45^{\circ} \mathrm{C}$ were used, and the result indicated that HIPEC at $44^{\circ} \mathrm{C}$ for $1.5 \mathrm{~h}$ had no significant effect on the mice vital signs, liver and renal function, but resulted in mild damage to the liver, kidney, small intestine and other organs. However, at $45^{\circ} \mathrm{C}$, HIPEC for $1.5 \mathrm{~h}$ had a marked impact on the animal's vital signs, resulting in serious damage to liver and kidney function, and notable pathological injuries to the liver, kidney, small intestine and other organs. To adjust for the difference in heat tolerance between humans and animals and the safety of clinical procedures, $43^{\circ} \mathrm{C}$ was selected as the temperature for hyperthermia therapy.

According to the above-mentioned results, the L-OHP concentration of $80 \mu \mathrm{g} / \mathrm{ml}$ and the optimum temperature of $43^{\circ} \mathrm{C}$ were selected to treat each group of cells. Results of the MTS assay demonstrated that, compared with the cell viability in the control group, cell viabilities in the HT, chemotherapy and chemotherapy + HT groups were significantly decreased $(\mathrm{P}<0.05)$, with the chemotherapy + HT group exhibiting the largest difference. The combined effect of L-OHP and hyperthermia was evaluated by the calculation of the q-value and the q-value was 1.370. The experimental results further verified the synergistic effect of L-OHP and HT. In addition, the cell 
growth in each group was observed under an inverted microscope. Compared with cells in the control group, cells in the hyperthermia and chemotherapy groups exhibited significantly lower density and marked rounded morphology $(\mathrm{P}<0.05)$. Among all the groups, cells in the chemotherapy + HT group demonstrated the slowest growth and the highest level of cell death, indicated by the majority of the cells becoming rounded and floating in the media. The results demonstrated synergistic cytotoxicity between HT and chemotherapy, and the combined therapy improved the L-OHP sensitivity in tumor cells, suggesting a clinical application for a reduced dose of chemotherapeutic agents in order to decrease short- and long-term side-effects, but also to maintain a cytotoxic effect with HT exposure.

ROS are a class of oxygen-containing compounds with potent biological activities generated by exogenous oxidants, or from intracellular aerobic metabolism. In health, ROS production and clearance is maintained in a dynamic balance. When ROS production exceeds clearance and intracellular antioxidants cannot effectively degrade and remove them, ROS accumulate in cells, resulting in oxidative stress $(19,20)$. This alters the opening of mitochondrial membrane channels by oxidizing unsaturated fatty acids in mitochondrial and cell membranes, resulting in increased membrane permeability (21). The increase in membrane permeability reduces the mitochondrial ATP and $\mathrm{Ca}^{2+}$ contents, which in turn results in the release of cytochrome $c$ and mitochondrial swelling. Studies suggest that severe damage to the mitochondria results in autophagy, autophagic cell death, apoptosis and necrosis $(22,23)$. ROS-induced tumor cell apoptosis is important in the association between ROS and apoptosis. Studies have demonstrated that ROS is associated with various types of apoptosis (21-23). In the present study, flow cytometry was used to detect intracellular ROS levels following treatment in each group of cells, and it was observed that the level of ROS was significantly higher in cells in the thermo-chemotherapy group than that in cells in the control group $(\mathrm{P}<0.05)$. Furthermore, using flow cytometry to assess rates of cell apoptosis in each group, it was observed that the proportion of apoptotic cells in the chemotherapy + HT group was significantly higher than in the chemotherapy, hyperthermia, and control groups $(\mathrm{P}<0.05)$, suggesting that thermo-chemotherapy induces apoptosis in SGC-7901 cells. The apoptosis may be mediated by ROS, and the current study hypothesizes that thermo-chemotherapy induces an accumulation of ROS in SGC-7901 cells, and the consequent oxidative stress induces tumor cell apoptosis and may be one of the major mechanisms that underlie the anti-tumor effects of thermo-chemotherapy.

Increasing evidence has demonstrated that ROS are involved in cell proliferation, differentiation and apoptosis, but also act as signaling molecules in the process of autophagy activation $(24,25)$. The process of autophagy is often accompanied by changes in ROS levels. Studies have observed that when autophagy is induced by therapeutic agents, the intracellular ROS levels change at the same time. For example, when lipopolysaccharides and the pan-caspase inhibitor, Z-VAD induce autophagy in macrophages, ROS levels in macrophages increase at the same time $(22,23,26,27)$. Studies have demonstrated that excessive accumulation of ROS leads to cytotoxic effects, which induce excessive autophagy and lead directly to autophagic cell death $(22,23,27)$. Beclin 1 and LC3, the autophagy markers, are involved in the process of autophagy. Quinsay et al (28) identified that the expression of Beclin 1 and LC3 is associated with ROS, and peroxides, a type of ROS, induce autophagy by upregulating Beclin 1 and LC3. Few studies have investigated the association between thermo-chemotherapy and autophagy. In order to further assess whether HT and chemotherapy-induced apoptosis is associated with autophagy, the formation of autophagic bodies were observed in each group by transmission electron microscopy. In cells of the chemotherapy and chemotherapy + HT groups, chromatin condensation and increased autophagic bodies were observed, and in the thermo-chemotherapy group, cells exhibited incomplete cell membranes and appeared close to cell death, indicating an association between autophagy and the cytotoxicity of L-OHP and HT in SGC-7901 cells.

Following the observation of autophagy by transmission electron microscopy, the underlying molecular mechanisms of autophagy induction by L-OHP or HT were investigated. When autophagy is induced, the expression of Beclin 1 increases markedly. Beclin 1 promotes the process of autophagy by serving as a "platform", forming complexes with a variety of proteins, and allowing the relocalization of autophagic proteins to autophagosomal membranes. mTOR, as a sensor of ATP, amino acids, growth factors and insulin, is important in the regulation of cell growth. mTOR inhibits autophagy by regulating the upstream and downstream signaling pathways, acting as a cell "doorman". Therefore, the present study focused on autophagy-associated proteins, LC3B, Beclin 1 and mTOR, for the investigation of the underlying mechanisms.

The current study investigated the mechanism of thermo-chemotherapy-induced cytotoxicity. Thermo-chemotherapy increased the level of intracellular ROS, and decreased the MMP, thereby reducing cell viability and inducing apoptosis. To assess whether chemotherapy, HT or thermo-chemotherapy-induced apoptosis is associated with autophagy, intracellular structures were observed using transmission electron microscopy. It was observed that, following chemotherapy or thermo-chemotherapy, a large number of autophagic bodies were produced, particularly in the thermo-chemotherapy group, where the highest number were observed, demonstrating that the L-OHP and HT result in autophagic cell death, and thermo-chemotherapy has the greatest cytotoxic effect. The molecular mechanisms of thermo-chemotherapy-induced cytotoxicity were also investigated. Western blot analysis demonstrated changes in protein levels of autophagy-associated genes, LC3B, Beclin 1 and $\mathrm{mTOR}$, in each group of cells, with the change in the chemotherapy + HT group being the most marked, suggesting that chemotherapy or thermo-chemotherapy-induced cell death is mediated by autophagy, and thermo-chemotherapy exerts an increased effect. The results suggest that thermo-chemotherapy may upregulate the expression of autophagy-associated proteins, Beclin 1 and LC3B via ROS, and induce autophagy in SGC-7901 human gastric cancer cells, leading to autophagic cell death. This process may be one of the major underlying mechanisms of the anti-tumor effects of thermo-chemotherapy.

The finding that ROS affects the level of autophagy is important for cancer research and therapeutic strategies. 
Although ROS were observed to be signaling molecules involved in the regulation of autophagy, the specific mechanisms by which they induce autophagy and the complete signal transduction pathways remain to be elucidated. In addition, whether various ROS work together or individually to regulate autophagy remains to be determined. The association between the ROS-induced autophagy-associated cell survival and cell death requires further investigation.

In conclusion, ROS production in gastric cancer cells downregulates the expression of the autophagic gene, m-TOR, and upregulates the expression of genes, Beclin 1 and LC3B. This indicates a positive correlation between ROS levels and the level of autophagy in gastric cancer cells. There is a linear association between thermo-chemotherapy-induced production of ROS and the cytotoxic effect of thermo-chemotherapy in gastric cancer cells, indicating an important role of thermo-chemotherapy-induced ROS production in autophagic cell death in gastric cancer cells. The consequent ROS-induced autophagic cell death is important to the cytotoxic effect of thermo-chemotherapy. However, the specific underlying mechanism of ROS accumulation and the occurrence of autophagy remain to be elucidated. In the future, further investigation into the specific mechanisms, and use of various types of cell lines, will indicate whether the results are consistent across different types of cancer.

\section{Acknowledgements}

The present study was supported by grants from the Guangdong Province and Hong Kong SAR Breakthrough Funds in Key Areas of Science and Technology (grant no. 2006Z1-E6041) and the Guangdong Provincial Science and Technology Program (grant no. 2009A030301013).

\section{References}

1. Tang Y,Liu X, Su B, Zhang Z, Zeng X, Lei Y, Shan J, Wu Y, Tang H and Su Q: microRNA-22 acts as a metastasis suppressor by targeting metadherin in gastric cancer. Mol Med Rep 11: 454-460, 2015.

2. Li H, Lu P, Lu Y, Liu C, Xu H, Wang S and Chen J: Predictive factors of lymph node metastasis in undifferentiated early gastric cancers and application of endoscopic mucosal resection. Surg Oncol 19: 221-226, 2010

3. Kato M, Ono S, Mabe K, Sakamoto N and Aaska M: After endoscopic treatment of early stage gastric cancer. Nihon Rinsho 71: 1429-1435, 2013 (In Japanese).

4. Valle M, Van der Speeten K and Garofalo A: Laparoscopic hyperthermic intraperitoneal peroperative chemotherapy (HIPEC) in the management of refractory malignant ascites: A multi-institutional retrospective analysis in 52 patients. J Surg Oncol 100: 331-334, 2009.

5. Ba MC, Cui SZ, Lin SQ, Tang YQ, Wu YB, Wang B and Zhang XL: Chemotherapy with laparoscope-assisted continuous circulatory hyperthermic intraperitoneal perfusion for malignant ascites. World J Gastroenterol 16: 1901-1907, 2010.

6. Kang R and Tang D: Autophagy in pancreatic cancer pathogenesis and treatment. Am J Cancer Res 2: 383-396, 2012.

7. Jabs T: Reactive oxygen intermediates as mediators of programmed cell death in plants and animals. Biochem Pharmacol 57: 231-245, 1999.

8. Li ZY, Yang Y, Ming M and Liu B: Mitochondrial ROS generation for regulation of autophagic pathways in cancer. Biochem Biophys Res Commun 414: 5-8, 2011.
9. Lehmann K, Rickenbacher A,Jang JH,OberkoflerCE, Vonlanthen R, von Boehmer L, Humar B, Graf R, Gertsch P and Clavien PA: New insight into hyperthermic intraperitoneal chemotherapy: Induction of oxidative stress dramatically enhanced tumor killing in in vitro and in vivo models. Ann Surg 256: 730-737, 2012

10. Chen F, Wang CC, Kim E and Harrison LE: Hyperthermia in combination with oxidative stress induces autophagic cell death in HT-29 colon cancer cells. Cell Biol Int 32: 715-723, 2008.

11. Law AS and Burt DW: qValue - a program to calculate comparative measures of genomic reorganisation from cytogenetic and/or linkage information. Comput Appl Biosci 12: 181-183, 1996.

12. Peng $\mathrm{J}$ and Wang Y: Epidemiology, pathology and clinical management of multiple gastric cancers: A mini-review. Surg Oncol 19: e110-e114, 2010.

13. Al-Shammaa HA, Li Y and Yonemura Y: Current status and future strategies of cytoreductive surgery plus intraperitoneal hyperthermic chemotherapy for peritoneal carcinomatosis. World J Gastroenterol 14: 1159-1166, 2008.

14. Liu YP, Ling Y, Qi QF, Zhang YP, Zhang CS, Zhu CT, Wang MH and Pan YD: Genetic polymorphisms of ERCC1-118, XRCC1-399 and GSTP1-105 are associated with the clinical outcome of gastric cancer patients receiving oxaliplatin-based adjuvant chemotherapy. Mol Med Rep 7: 1904-1911, 2013.

15. Zhang XL, Shi HJ, Cui SZ, Tang YQ and Ba MC: Prospective, randomized trial comparing 5-FU/LV with or without oxaliplatin as adjuvant treatment following curative resection of gastric adenocarcinoma. Eur J Surg Oncol 37: 466-472, 2011.

16. Liu JF, Zhou XK, Chen JH, Yi G, Chen HG, Ba MC, Lin SQ and Qi YC: Up-regulation of PIK3CA promotes metastasis in gastric carcinoma. World J Gastroenterol 16: 4986-4991, 2010.

17. Ba M, Cui S, Lin S, Tang Y, Wu Y and Zhang X: Resection of a giant hepatocellular carcinoma weighing over ten kilograms. World J Gastroenterol 16: 1422-1424, 2010.

18. Cui S, Ba M, Huang D, Tang Y and Wu Y: Study and development of BR-TRG-I hyperthermic perfusion intraperitoneal treatment system. China Medical Devices 24: 7-9, 2009.

19. Fleury C, Mignotte B and Vayssière JL: Mitochondrial reactive oxygen species in cell death signaling. Biochimie 84: $131-141,2002$.

20. Thannickal VJ and Fanburg BL: Reactive oxygen species in cell signaling. Am J Physiol Lung Cell Mol Physiol 279: $1005-1028,2000$.

21. Cui K, Luo X, Xu K and Ven Murthy MR: Role of oxidative stress in neurodegeneration: Recent developments in assay methods for oxidative stress and nutraceutical antioxidants. Prog Neuropsychopharmocol Biol Psychiatry 28: 771-799, 2004.

22. Lemasters JJ, Nieminen AL, Qian T, Trost LC, Elmore SP, Nishimura Y, Crowe RA, Cascio WE, Bradham CA, Brenner DA and Herman B: The mitochondrial permeability transition in cell death: A common mechanism in necrosis, apoptosis and autophagy. Biochim Biophys Acta 1366: 177-196, 1998

23. Vurusaner B, Poli G and Basaga H: Tumor suppressor genes and ROS: Complex networks of interactions. Free Radic Biol Med 52: 7-18, 2012.

24. Scherz-Shouval R and Elazar Z: Regulation of autophagy by ROS: Physiology and pathology. Trends Biochem Sci 36: 30-38, 2011.

25. Scherz-Shouval R, Shvets E, Fass E, Shorer H, Gil L and Elazar Z: Reactive oxygen species are essential for autophagy and specifically regulate the activity of Atg4. EMBO J 26: 1749-1760, 2007.

26. Xu Y, Kim SO, Li Y and Han J: Autophagy contributes to caspase-independent macrophage cell death. J Biol Chem 281: 19179-19187, 2006.

27. Liu HD and Lu JX: Progress in mitophagy. Chin J Cell Biol (Henderson NV) 23: 467-471, 2008.

28. Quinsay MN, Thomas RL, Lee Y and Gustafsson AB: Bnip3-mediated mitochondrial autophagy is independent of the mitochondrial permeability transition pore. Autophagy 6 : $855-862,2010$ 